\title{
INFLUENCE OF HUMIC SUBSTANCES ON PHOTOLYSIS OF NITROAROMATIC COMPOUNDS IN AQUEOUS SYSTEMS
}

\author{
Milagros S. Simmons ${ }^{1}$ and Richard G. Zepp ${ }^{2}$ \\ 'Department of Environmental and Industrial Health, The University of Michigan, Ann Arbor, MI 48109 \\ and ${ }^{2}$ Environmental Research Laboratory, U.S. Environmental Protection Agency, Athens, GA 30613, \\ U.S.A.
}

(Received September 1985)

\begin{abstract}
Results are reported for kinetic studies of the photolysis of 19 nitroaromatic compounds in water. The results indicate that dissolved humic substances in natural waters enhance the sunlight-induced photodegradation rates of nitrobenzenes, nitrotoluenes, and nitroxylenes compared to rates observed in distilled water. The largest enhancements, ranging from 2 to 26 -fold, were observed for nitroaromatics that are methylated ortho to the nitro group. Similar enhancement effects were observed for humic substances obtained from a variety of terrigenous sources, including humus in natural waters and humus extracted from soils.
\end{abstract}

Key words - nitroaromatics, humic substances, aquatic photochemistry, quantum yields, nitrotoluenes, nitrobenzenes, nitroxylenes, sunlight photolysis

\section{INTRODUCTION}

Nitroaromatic compounds are present in a variety of products, including pesticides and explosives. Chemicals in this family have been detected in ambient freshwater and marine environments, drinking water supplies and industrial effluents (Shackelford and Keith, 1976; Hashimoto et al., 1982; Spanggord et al., 1980). Three of the compounds considered in the present study, nitrobenzene, 2,4-dinitrotoluene and 2,6-dinitrotoluene, are listed as priority pollutants in Section 307(a) (1) of the 1977 Clean Water Act. Others are structurally related to important pesticides such as methyl parathion (O,O-dimethyl-O $p$-nitrophenyl phosphorothioate) and lampricide (3-trifluoromethyl-4-nitrophenol).

Various laboratory and field investigations have shown that certain nitroaromatic compounds are readily transformed on entry into aquatic environments (Mabey et al., 1983; Callahan et al., 1979). Because nitroaromatic compounds absorb sunlight strongly in the ultraviolet and blue spectral region, they are generally susceptible to photochemical transformation in aquatic systems. For example, field studies by Carey and Fox (1981) of lampricide and by Mabey et al. (1983) of trinitrotoluene have shown that photoreactions dominate the behavior of some nitroaromatics in streams and rivers. Other studies also have shown that the environmental photoreactivity of nitroaromatic chemicals varies considerably, being strongly dependent on molecular structure as well as on the natural constituents of aquatic environments (Spanggord et al., 1980; Mabey et al., 1983).

The sometimes-dramatic influence of natural substances on photolysis rates of nitroaromatic com- pounds is illustrated by studies of Mabey et al. (1983). These workers found that the photolysis of trinitrotoluene was accelerated up to 100-fold in natural waters compared to pure water. Zepp and Baughman (1978) and Mabey et al. (1983) reported that the enhancement, or "sensitization", of photolysis rates of the nitroaromatics methyl parathion and trinitrotoluene in natural waters is at least in part caused by humic substances in the water. Other studies have shown that humic substances are capable of photosensitizing several types of organic reactions (Mill et al., 1980; Zepp et al., 1985), but little is known about the general susceptibility of nitroaromatics to these humus-induced photoreactions.

The objectives of this study were to first determine how variations in the structure of monocyclic nitroaromatic compounds affect their direct photoreaction rates in distilled water. Then, through studies in sunlight and under artificial light, the influence of various humic substances on photoreactions of these nitroaromatics are determined by comparing results in humus-containing water with those in distilled water. The investigation provides kinetic data, such as reaction quantum yields, and equations that can be used to assess the degradation of nitroaromatic pollutants in a variety of aquatic environments. In addition, information is provided about how natural substances influence the photochemical transformation of chemicals in aquatic systems.

\section{EXPERIMENTAL.}

\section{Reagents}

The water used was doubly distilled; the last distillation was over permanganate solution to obtain organic-free water. The nitroaromatics were purchased from Aldrich 
Chemical Co. (Milwaukee. Wis.) except for the 2,6-dinitrotoluene and the 2,4-dinitrotoluene, which were obtained as standard reference materials from the U.S Environmental Protection Agency (Research Triangle Park, N.C.). Saturated solutions of the nitroaromatics were made up in distilled water and were centrifuged at $15,000 \mathrm{rpm}$ for $30 \mathrm{~min}$. The supernatant was removed carefully and this stock solution was diluted as described below. Solutions of the nitroaromatics in acetonitrile (Burdick \& Jackson Laboratories, Muskegon, Mich.) were used for the stock standards. Dilutions of the stock standards in water were used as working standards.

Water samples that were collected from the top meter of highly colored rivers in Georgia and Florida were kep refrigerated at $4 \mathrm{C}$. Fulvic acid was obtained commercially from Contech ETC, Ottawa, Canada. A fulvic acid isolated from Wylde Lake, Ontario, Canada, was obtained as a gift from Dr John Carey, Canada Centre for Inland Waters. Dr E. M. Perdue, Georgia Institute of Technology. provided a fulvic acid isolated from the Altamaha River in Georgia

p-Nitroanisole was obtained from Aldrich Chemical Co. Acetonitrile used as the solvent system for the liquid chromatography was obtained from Burdick \& Jackson Laboratories.

Apparatus

The aqueous nitroaromatic stock solutions were centrifuged with a Sorval ultracentrifuge. The humus solutions were filtered using a Millipore filtering apparatus. The absorbances of all the aqueous nitroaromatic solutions and the humus solutions were measured with a Perkin-Elmer Lambda 3 u.v.-visible spectrophotometer. Organic carbon concentrations in the humic solutions were analyzed with a Beckman Model 915 organic carbon analyzer. The disappearances of the parent compound and the actinometer solutions were followed using a Micromeritics high pressure liquid chromatograph (HPLC) equipped with a Whatman Partisil PXS 10/25 ODS-2 column. Ultraviolet absorption spectra were obtained using a Perkin-Elmer Lambda 3 spectrophotometer

A merry-go-round photoreactor (Moses et al., 1969) equipped with a $450 \mathrm{~W}$ mercury lamp and Corning $0-52$ and $7-37$ filters to isolate the $366-\mathrm{nm}$ line was used for the monochromatic light exposures. A $1-\mathrm{cm}$ thick solution of $0.001 \mathrm{M}$ potassium chromate in $3 \%$ potassium carbonate was used in the merry-go-round to isolate the $313-\mathrm{nm}$ line

\section{Procedures}

The saturated aqueous solutions of nitroaromatics were diluted to concentration levels of $10^{-6}-10^{-5} \mathrm{M}$ in distilled water, natural waters and aqueous solutions of extracted natural humic materials for use in the experiments. The humus solutions were spectrally matched at different u.v wavelengths prior to addition of the substrate.

Triplicate solutions in Pyrex test tubes were exposed to mid-day sunlight (Athens, Ga) and monochromatic light $(313$ and $366 \mathrm{~nm})$. Exposures with monochromatic light were accomplished in the merry-go-round photoreactor which uniformly irradiates all the solutions at the same time. An aqueous solution of $p$-nitroanisole $\left(10^{-6} \mathrm{M}\right)$ was used as actinometer for both sunlight and monochromatic light exposures to measure the light intensity during irradiation (Dulin and Mill, 1982; Zepp et al., 1985). The exposure times were varied for the different nitroaromatics, achieving approx. 30\% reaction for each exposure. Dark controls were used in each run. The solutions were then analyzed by reverse phase HPLC using 40-80- $\mu$ l sample injections with acetonitrile-water solvent system $(70: 30)$ at a flow-rate of $1.5 \mathrm{ml} \mathrm{min}{ }^{-1}$. Dark controls were analyzed similarly. Dark controls showed no transformation during the periods required for the experiments, which in most cases were less than 1 day.

The disappearance rate constant $\left[k_{n}(P)\right]$ of the parent nitroaromatic compound was calculated as follows

$$
k_{\prime \prime}(P)=\frac{1}{t} \times \ln \left(\begin{array}{c}
P_{0} \\
P_{t}
\end{array}\right)
$$

where $P_{0}$ is the concentration of the nitroaromatic compound at initial time, and $P$ is the concentration of the compound at the end of exposure time $(t)$, expressed in hours. The $k_{p}$ for the $p$-nitroanisole $\left[k_{p}(\mathrm{PNA})\right]$ was calculated in a similar manner. Reaction quantum yields $\left(\phi_{r}\right)$ were computed from the kinetics data obtained using monochromatic light (Zepp, 1978, 1982). For the sunlight experiments, the rate constants were normalized to the actinometer rate constants [see equation (2)] [Dulin and Mill, 1982) to correct for variations in light intensity.

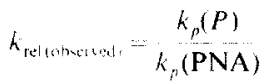

Because humic substances strongly absorb light in the u.v. region, rate constants in the humic solutions were reduced by light attenuation effects. These light attenuation effects may be quantitated using a light screening factor, $S$ (Zepp et al, 1985) where $S$ is defined as the ratio of the photolysis rate constant in a system with light attenuation to the rate constant in a system with no attenuation. This factor was computed using the following equation, which assumes complete mixing.

$$
S=\frac{1-10}{2.303 A}
$$

where $A$, is the absorbance of the solution at the wavelength used in the experiment. To compute $S$ for the sunlight experiments, the absorbances of the humus solutions at $330 \mathrm{~nm}$ were used. This wavelength represents the region of the spectrum at which the nitroaromatics absorb solar radiation most rapidly.

Rate constants for photoreactions in an infinitesimally thin layer of humus solution, $k_{\text {surf }}$, were computed as follows.

$$
k_{\text {surf }}=\frac{k_{\text {rel (observed) }}}{\mathrm{S}}
$$

where $k_{\text {surf }}$ represents the disappearance rate constant incorporating both corrections for variation in light intensity during exposure as well as light attenuation effects by the humus solutions.

\section{RESULTS AND DISCUSSION}

\section{Direct photolysis in pure water}

The nitroaromatic compounds included in this study all exhibit similar broad absorption spectra extending from the u.v. into the short-wavelength visible region. These spectra strongly overlap the spectrum of ground-level sunlight. Because photolysis through direct absorption of sunlight is generally rapid for chemicals that strongly absorb sunlight (Zepp and Cline, 1977), the direct photolysis of several nitroaromatic compounds was studied in detail.

Direct photolysis was studied in distilled water to examine the influence of structural variations on the photoreactivity of substituted nitroaromatic compounds. In distilled water, direct photolysis is the only available photochemical pathway and equation (5) describes the direct photolysis rate $(-\mathrm{d}[P] / \mathrm{d} t)$ of a nitroaromatic compound in a weakly absorbing solution (Zepp, 1982).

$$
-\frac{\mathrm{d}[P]}{\mathrm{d} t}=k_{a} \phi_{r}[P]=k_{n}[P]
$$


Table 1. Kinetic data for direct photolysis of selected nitroaromatic compounds in water

\begin{tabular}{|c|c|c|c|c|}
\hline Compound & $\begin{array}{c}\text { Reaction quantum } \\
\text { yield, } \phi_{r}\end{array}$ & $\begin{array}{c}\text { Wavelength } \\
(\mathrm{nm})\end{array}$ & $\begin{array}{c}\text { Typical } \\
\text { near-surface } k_{\mathrm{a}} \\
\left.\text { (days }^{-1}\right)^{*}\end{array}$ & $\begin{array}{c}\mathbf{k}_{\mathrm{a}} \boldsymbol{\phi}_{r} \\
\left(\text { days }^{-1}\right)\end{array}$ \\
\hline I. Nitrobenzene & $(2.9 \pm 1.0) \times 10^{-5}$ & 313 & 178 & $5.2 \times 10^{-3}$ \\
\hline 2. 2-Nitrotoluene & $(2.2 \pm 0.4) \times 10^{3}$ & 366 & 399 & $8.8 \times 10^{1}$ \\
\hline 3. 3-Nitrotoluene & $(2.1 \pm 0.4) \times 10^{2}$ & 366 & 310 & 6.5 \\
\hline 4. 4-Nitrotoluene & $(5.2 \pm 0.86) \times 10^{3}$ & 366 & 537 & 2.8 \\
\hline 5. 2-(Trifluoromethyl)nitrobenzene & $(2.3 \pm 0.51) \times 10^{-4}$ & 313 & 214 & $4.9 \times 10^{-2}$ \\
\hline 6. 3-(Trifluoromethyl)nitrobenzene & $(1.9 \pm 0.48) \times 10^{-4}$ & 313 & 88 & $1.7 \times 10^{2}$ \\
\hline 7. 4-(Trifluoromethyl)nitrobenzene & $(2.3 \pm 0.51) \times 10^{4}$ & 313 & 95.2 & $2.2 \times 10^{2}$ \\
\hline 8. 2-Nitro-1,3-xylene & $(2.3 \pm 0.5) \times 10^{3}$ & 366 & 254 & $5.8 \times 10^{1}$ \\
\hline 9. 4-Nitro-1,3-xylene & $(1.4 \pm 0.3) \times 10^{-3}$ & 366 & 705 & $9.9 \times 101$ \\
\hline 10. 5-Nitro-1,3-xylene & $(6.4 \pm 1.1) \times 10^{3}$ & 366 & 577 & 3.7 \\
\hline 11. 4-Nitro-1,2-xylene & $(1.1 \pm 0.2) \times 10^{1}$ & 366 & 969 & $1.1 \times 10^{2}$ \\
\hline 12. 2,4,6-Trifluoronitrobenzene & $(4.5 \pm 0.99) \times 10^{-4}$ & 313 & 290 & $1.3 \times 10^{\prime}$ \\
\hline 13. 1,3-Dinitrobenzene & $(1.6 \pm 0.4) \times 10^{-4}$ & 313 & 189 & $3.0 \times 10^{-2}$ \\
\hline 14. 2,6-Dinitrotoluene & $(1.2 \pm 0.2) \times 10^{-3}$ & 366 & 557 & $6.7 \times 10^{-1}$ \\
\hline 15. 2,4-Dinitrotoluene & $(2.0 \pm 0.47) \times 10^{-3}$ & 313 & 358 & $7.2 \times 10^{-1}$ \\
\hline 16. 2,3-Dinitrotoluene & $(7.0 \pm 1.2) \times 10^{4}$ & 366 & 506 & $3.5 \times 10^{-1}$ \\
\hline 17. 3,4-Dinitrotoluene & $(7.2 \pm 1.8) \times 10^{-5}$ & 366 & 723 & $5.2 \times 10^{-2}$ \\
\hline 18. 2,5-Dinitrotoluene & $(1.0 \pm 0.16) \times 10^{-2}$ & 366 & 896 & 9.0 \\
\hline 19. 4-Nitroanisole & $(3.2 \pm 0.5) \times 10^{-4}$ & 320 & 3180 & 1.02 \\
\hline
\end{tabular}

*Computed by averaging over a full year at latitude $40^{\circ} \mathrm{C}$.

where

$$
k_{a}=\int E_{0} \epsilon_{\lambda} d \lambda
$$

Two key parameters are required to compute the direct photolysis rate constant $\left(k_{\mathrm{p}}\right)$ - the specific light absorption rate $\left(k_{a}\right)$ and the reaction quantum yield $\left(\phi_{r}\right)$. The light absorption rate is a measure of the spectral overlap between the irradiance $\left[E_{0}(\lambda)\right]$ and the molar absorptivity $\left(\epsilon_{\lambda}\right)$ of the nitroaromatic compound, integrated over the photoactive wavelengths of the light [equation (6)]. The reaction quantum yield is the fraction of absorbed light that results in chemical reaction.

Kinetic results for several monocyclic nitroaromatic compounds are summarized in Table 1. All results pertain to very dilute solutions $(1 \mu \mathrm{m})$ of the nitroaromatic compounds in pure, air-saturated water. The specific sunlight absorption rates were calculated using the GCSOLAR computer program, a revised version of a program that was described by Zepp and Cline (1977). Molar absorptivities for the nitroaromatic compounds in pure water were used as inputs for these computations. The variation in substituents on the nitroaromatic ring resulted in changes in the sunlight absorption rates. In general, electron releasing substituents, e.g. $-\mathrm{CH}_{3}$ and $-\mathrm{OCH}_{3}$, enhanced $k_{a}$ compared to nitrobenzene itself whereas electron withdrawing substituents, e.g. $-\mathrm{NO}_{2}$ and $-\mathrm{CF}_{3}$, had little effect or slightly decreased $k_{u}$.

The changes in light absorption rates caused by substituent variation were minor compared to the changes in reaction quantum yields: quantum yields varied over a range of about four orders of magnitude whereas changes in $k_{a}$ ranged over about one order of magnitude (Table 1). Generally, the photoreactions were very inefficient, usually with quantum yields less than $1 \%$. Examination of Table 1 leads to several conclusions concerning the relationship between structure and reaction quantum yields for the nitroaromatics:

(1) Substitution for hydrogen by $-\mathrm{CF}_{3},-\mathrm{NO}_{2}$, $-\mathrm{OCH}_{3}$ and $-\mathrm{CH}_{3}$ on the nitrobenzene ring enhanced the quantum efficiency for reaction. Nitrobenzene was the most inefficient of all compounds studied.

(2) Substitution by methyl groups particularly enhances the photolysis efficiency of the nitroaromatic system. For example, the nitrotoluenes are 2 to 3 orders of magnitude more efficient than nitrobenzene.

(3) Substitution adjacent to the nitro group tends to decrease quantum efficiencies. The most dramatic example of this apparent steric effect occurred with the dinitrotoluenes. The compounds with adjacent nitro groups (2,3- and 3,4-dinitrotoluene) were considerably less reactive than the other dinitrotoluenes included in the study. Also, 2-nitrotoluene photoreacted less efficiently than the other nitrotoluenes.

(4) The reaction quantum yields of the nitrotoluenes were greatly decreased by replacement of the methyl hydrogen atoms by the more strongly bonded fluorine atoms.

(5) Two adjacent methyl substituents on the aromatic ring produced a remarkable enhancement in quantum yield (see 4-nitro-1,2-xylene compared to the other nitroxylenes).

A few comments should be made concerning the above conclusions. First, the reaction quantum yields of some nitroaromatic compounds are known to be susceptible to $\mathrm{pH}$ effects (Cornelisse et al., 1979), at least in the basic region. The results in Table 1 were all obtained at pH 5.5. Moreover, the reader is cautioned that reaction quantum yields may have little or no relationship to excited state reaction rates. Chemical reaction of an excited state is but one of several factors that determine the reaction quantum yield. Other important factors include the decay of the excited state back to ground state, the efficiency 


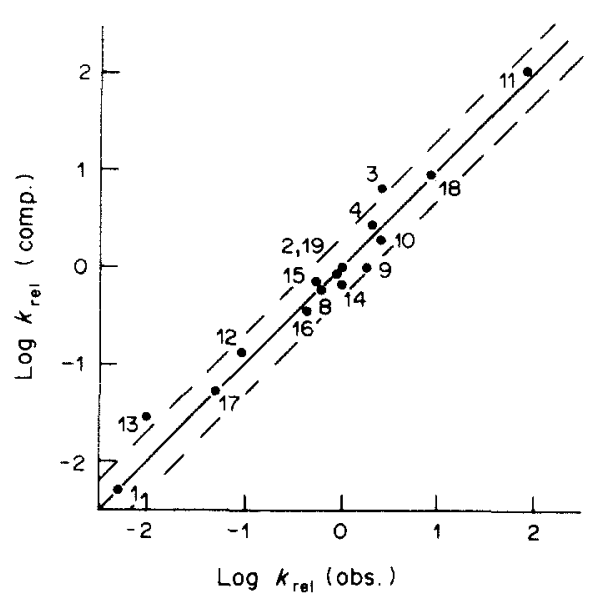

Fig. 1. Comparison of sunlight photolysis rate constants computed using laboratory data (Table 1) and determined from outdoor experiments. Numbers correspond to nitroaromatic compounds listed in Table 1; dashed line indicates factor of 2 from exact agreement (solid line).

of photoproduction of the excited state that reacts, and the reversion of unstable photoproducts back to starting material. Efforts to disentangle the effects of structural changes on these various processes were beyond the scope of this study.

Despite the low quantum efficiencies of the nitroaromatic photoreactions, the photolysis of some of these compounds under full exposure to sunlight is rapid. Computations of average annual rate constants (Table 1) using GCSOLAR program (Zepp and Cline, 1977) indicate that the nitroaromatic compounds substituted by methyl or methoxyl had photolysis half-lives $\left(0.693 / k_{a} \phi_{r}\right)$ that are less than 1 day. On the other hand, nitrobenzene and the nitroaromatics with electron withdrawing substituents, because of their lower quantum yields and sunlight absorption rates, photoreact much more slowly (half-lives on the order of weeks to months). The computations in the table are averaged annual values that pertain to near-surface conditions at latitude $40^{\circ} \mathrm{N}$. Variations in rate constants caused by changes in season, latitude, and water depth are discussed by Zepp and Cline (1977).

Experiments also were carried out in sunlight at Athens, Ga (latitude $34 \%$ ) to determine $k_{\text {rel (observed) }}$ [see equation (2)], the sunlight photolysis rate constant ratioed to the rate constant for the $p$ nitroanisole actinometer. These experimental values agreed satisfactorily with theoretical values, $\mathrm{k}_{\text {rel(computed), }}$ that were calculated using the data in the last column of Table 1 (Fig. 1).

$$
k_{\text {rel (compuled })}=\frac{k_{a} \phi_{r}(P)}{k_{a} \phi_{r}(\mathrm{PNA})} \text {. }
$$

In equation (7), $k_{u} \phi_{r}(P)$ corresponds to the nitroaromatic compound and $k_{u} \phi_{r}$ (PNA) corresponds to $p$-nitroanisole. Both experiment and theory showed that the value of $k_{\text {rel }}$ remained constant with changes in sunlight intensity that result from cloud cover and seasonal changes. The $p$-nitroanisole actinometer and the theoretical basis for its usage are discussed in more detail by Dulin and Mill (1982).

\section{Effects of humic substances on photolysis of nitro- aromatic compounds}

Natural waters contain various substances that influence the rates of sunlight-induced reactions of pollutants. Past studies have focused mainly on effects of humic substances on photoreactions of pollutants that do not absorb sunlight (Mill et al., 1980; Zepp et al., 1985). Humic substances included in these studies were primarily terrigenous in origin, i.e. the colored organic matter derived from terrestrial plant decay that occurs in soils and in inland and coastal waters. These humic substances have been found to catalyze photoreactions by sensitizing photooxidations and other types of photoreactions. In a detailed study of 2,4,6-trinitrotoluene (TNT), Mabey et al. (1983) found that its photolysis was strongly accelerated in certain natural waters and in aqueous solutions of humic substances.

To investigate the generality of the effect of humic substances on photolysis of nitroaromatic compounds, the sunlight photolysis rate constants were determined for a series of nitroaromatics in solutions of humic substances from various sources. Because the earlier work by Mabey et al. (1983) had shown no $\mathrm{pH}$ effect on photolysis rates of TNT in natural waters, $\mathrm{pH}$ effects were not included in the studies. As in the case of the direct photolysis studies discussed above, the $p$-nitroanisole outdoor actinometer was used. The $k_{\text {ret }}$ values obtained in these experiments were slightly affected by light attenuation by the humic substances. To estimate near surface values, a correction was applied [equation (4)] assuming that the maximum effect of the sunlight occurs at about $330 \mathrm{~nm}$. This assumption, even if not exact, resulted in only about a $25 \%$ correction; the maximum light attenuation correction for these solutions would be only about $30 \%$.

In the first such study, 13 compounds were studied in a water sample from the Aucilla River, a colored river in north Florida, and in solutions of two fulvic acids, one isolated from Wylde Lake in Ontario. Canada, and the other isolated from a soil and sold by Contech ETC. The concentrations of the fulvic acids were adjusted so that the solutions optically matched the Aucilla River water. Zepp and Schlotzhauer (1982) provide a more detailed treatment of the spectral characteristics of humic substances. Results of this study showed that the humic substances enhanced the photolysis rates (compared to distilled water results) of most of the nitroaromatics that were studied.

The enhancements observed in the humus solutions were by far most pronounced in the case of six compounds: $\theta$-nitrotoluene, 2,6-dinitrotoluene, 2,4dinitrotoluene, 2-nitro-1,3-xylene, 4-nitro-1,3-xylene 
Table 2. Sunlight exposure studies of nitroaromatic compounds in various optically matched solutions containing humic substances*

\begin{tabular}{|c|c|c|c|c|}
\hline \multirow[b]{2}{*}{ Compound } & \multirow[b]{2}{*}{$k_{\mathrm{rcl}}\left(\mathrm{H}_{2} \mathrm{O}\right)$} & \multicolumn{3}{|c|}{$k_{\text {surf }}$ [ratio to $k_{\text {rel }}\left(\mathrm{H}_{2} \mathrm{O}\right)$ is parenthesized] } \\
\hline & & Aucilla River & $\begin{array}{c}\text { Contech } \\
\text { fulvic acid }\end{array}$ & $\begin{array}{l}\text { Wylde Lake } \\
\text { fulvic acid }\end{array}$ \\
\hline Nitrobenzene & 0.005 & $0.007(1.4)$ & $0.007(1.4)$ & - \\
\hline 2,4,6-Trifluoronit robenzene & 0.09 & $0.13(1.4)$ & $0.26(2.9)$ & $0.29(3.2)$ \\
\hline$m$-Dinitrobenzene & 0.01 & $0.029(2.9)$ & $0.043(4.3)$ & - \\
\hline$o$-Nitrotoluene & 0.88 & $6.6(7.5)$ & $5.6(6.4)$ & $5.5(6.2)$ \\
\hline$m$-Nitrotoluene & 2.6 & $3.1(1.2)$ & $2.7(1.1)$ & $2.7(1.1)$ \\
\hline$p$-Nitrotoluene & 2.0 & $2.4(1.2)$ & $2.4(1.2)$ & $2.4(1.2)$ \\
\hline 2,6-Dinitrotoluene & 0.98 & $17(17.3)$ & $15(15.3)$ & $11(11.0)$ \\
\hline 2,4-Dinit rotoluene & 0.54 & $2.5(4.6)$ & $2.5(4.6)$ & $1.3(2.4)$ \\
\hline 2- $\mathrm{NO}_{2}-1,3$-xylene & 0.66 & $1.3(2.0)$ & $1.3(2.0)$ & $0.92(1.4)$ \\
\hline 5- $\mathrm{NO}_{2}-1,3$-xylene & 2.5 & $2.1(0.9)$ & $2.9(1.2)$ & $1.9(0.8)$ \\
\hline $4-\mathrm{NO}_{2}-1,3$-xylene & 1.8 & $4.5(2.5)$ & $4.3(2.4)$ & $3.6(2.0)$ \\
\hline 4- $\mathrm{NO}_{2}-1,2$-xylene & 83.7 & $93(1.1)$ & $92(1.1)$ & $92(1.1)$ \\
\hline 3- $\mathrm{NO}_{2}-1,2$-xylene & 35.4 & $85(2.4)$ & $68(1.9)$ & $40(1.1)$ \\
\hline
\end{tabular}

*Absorbance $(1.0 \mathrm{~cm}$ pathlength) equalled 0.25 at $330 \mathrm{~nm}$.

+ Standard deviations are $\leqslant 25 \%$ of value shown for $k_{\text {rel }}$ or $k_{\text {surf }}$.

and 3-nitro-1,2-xylene. All of these compounds have one structural feature in common: a nitro group adjacent, i.e. ortho, to a methyl group on the aromatic ring. Compounds with two nitro groups flanking a methyl group - e.g. 2,6-dinitrotoluene and TNT - are particularly susceptible to such catalysis of their photolysis (Mabey et al., 1983). On the other hand, the photolysis rate of the simplest nitroaromatic, nitrobenzene, was hardly affected by the presence of humic substances.

The studies reported in Table 2 also indicated that humic substances isolated from soil or from a natural water body had very similar abilities to catalyze the nitroaromatic photoreactions. To further examine this point, additional studies were conducted with 2,6-dinitrotoluene (Table 3). Results of these studies indicated that the catalytic effects of natural substances in optically matched natural water samples from Georgia and Florida and in an aqueous solution of a riverine fulvic acid isolated from a Georgia river were remarkably similar. In all cases, the rate was enhanced by about one order of magnitude. This similarity strongly indicates that the non-humic or-

Table 3. Effect of various humic substances on photolysis rate of 2,6-dinitrotoluene in water*

\begin{tabular}{lccc}
\hline $\begin{array}{c}\text { Source of } \\
\text { humic substances } \dagger\end{array}$ & $\begin{array}{c}\text { TOC } \dagger \\
\left(\mathrm{mg} \mathrm{I}^{-1}\right)\end{array}$ & $\begin{array}{c}A_{330} 8 \\
\left(\mathrm{~cm}^{-1}\right)\end{array}$ & $k_{\text {surf }}$ \\
\hline Aucilla R., Fla & 9.6 & 0.197 & 9.8 \\
River Styx, Fla & 9.6 & 0.189 & 12 \\
Okefenokee Swamp, Ga & 9.1 & 0.208 & 11 \\
Suwannee R., Ga & 10.5 & 0.196 & 11 \\
Santa Fe R., Fla & 9.5 & 0.183 & 10 \\
Possum R., Fla & 10.0 & 0.193 & 14 \\
Fulvic acid isolated & 13.6 & 0.212 & 10 \\
$\quad \begin{array}{l}\text { from Altamaha R., Ga } \\
\text { In distilled water }\end{array}$ & & & 0.98 \\
\hline
\end{tabular}

*Based on exposure to sunlight at Athens, Ga, using $p$-nitroanisole actinometer.

tExcept as noted, all experiments conducted in river water samples that were diluted by distilled water.

$\ddagger$ Total organic carbon content of humus solution.

\$Absorption coefficient (decadic) of humus solution at $330 \mathrm{~nm}$.

q First order rate constant ratioed to rate constant for actinometer corrected for light attenuation [equation (4)]; standard deviation $\leqslant 25 \%$ of ratio. ganic components of the natural waters used here had no more than a minor effect on the nitroaromatic photochemistry.

Experiments also were conducted with 2,6-dinitrotoluene to determine the effects of varying the concentration of humic substances on the sunlight photolysis rate constant. Typical results of such experiments are shown in Fig. 2, which pertains to rate studies in water samples from the Aucilla River. The river water was diluted by distilled water to obtain various total organic carbon (TOC) concentrations. The results show that, within experimental error, there is a linear relationship between the photolysis rate constant and TOC.

Prior studies of the behavior of dinitrotoluene isomers in the seawater of Dokai Bay, Japan, indicated that an unidentified environmental process degraded 2,6-dinitrotoluene more rapidly than 2,4-dinitrotoluene (Hashimoto et al., 1982). Based on the results observed in this study, it seems likely that this degradation process is photochemical in nature. As results in Table 2 indicate, the photolysis of the 2,4- and 2,6-isomers of dinitrotoluene is rapid and is enhanced in natural waters. Moreover, consistent with the field observations of Hashimoto et al. (1982),

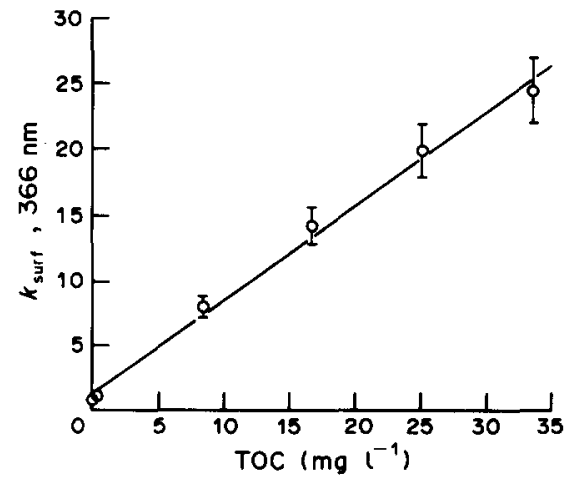

Fig. 2. Photolysis rate constants $(366 \mathrm{~nm})$ of 2,6 -dinitrotoluene in various dilutions of Aucilla River water. 
the 2,6-isomer is more photoreactive than the 2,4-isomer, especially with humic substances present in the water. Humic substances are a likely constituent of the bay water.

\section{CONCLUSIONS}

The studies reported here indicate that photolysis rates of nitroaromatic compounds are strongly dependent on the nature of the substituent on the nitroaromatic ring. This variability is mainly attributable to differences in the reaction quantum efficiencies of the compounds. Substitution of the aromatic ring with methyl groups greatly enhances photolysis rates, especially when humic substances are present in the water and the methyl group is ortho to the nitro group. The near surface half-lives of all of the methylated nitroaromatic compounds studied were found to be less than one day of average sunlight (latitude $40^{\circ} \mathrm{N}$ ). Direct photolysis rate constants observed for 17 nitroaromatics in outdoor experiments were found to be within a factor of two of those predicted by theory.

Humic substances enhanced the photolysis rates of the nitroaromatic compounds included in this study. The magnitude of the enhancement depended on the ring substituent of the nitroaromatic; compounds with methyl groups ortho to the nitro group were especially susceptible to this effect. Near-surface photolysis rates in the presence of humic substances were directly proportional to the concentration of the humic substance. Humic substances from a variety of terrigenous sources were found to have similar catalytic efficiencies.

The photolysis of dinitrotoluene isomers was found to be rapid in natural waters. The 2,6-isomer photolyzes more rapidly than the 2,4 -isomer. This finding is consistent with field studies in Dokai Bay, Japan, which showed that the 2,6-isomer is degraded more rapidly than the 2,4-isomer.

Acknowledgements-We thank the National Research Council for its partial funding of this research and $\mathrm{Dr}$ Lawrence Burns for his assistance in collecting some of the natural water samples.

\section{REFERENCES}

Callahan M. A., Slimak M. W., Gabel N. W., May I. P.,
Fowler C. F., Freed J. R., Jennings P., Durfee R. L., Whitmore F. C., Maestri B., Mabey W. R., Holt B. R and Gould C. (1979) Water-related environmental fate of 129 priority pollutants. EPA-440/4-79-029a. Washington. D.C.

Carey J. H. and Fox M. E. (1981) Photodegradation of the lampricide 3-trifluoromethyl-4-nitrophenol (TEM)--I Pathway of direct photolysis in solution. J. Great Lake's Res. 7, 234-241

Cornelisse J., Lodder G. and Havinga E. (1979) Pathways and intermediates of nucleophilic aromatic photosubstitution reactions. Rev. Chem. Intermed. 2, 231 265

Dulin D. and Mill T. (1982) Development and evaluation of sunlight actinometers. Envir. Sci. Technol. 16, 815-820.

Hashimoto A., Sakino H., Kojima T., Yamagami E., Tateishi $S$. and Akiyama T. (1982) Sources and behavior of dinitrotoluene isomers in sea-water. Wat. Res. 16 , 891-897.

Mabey W. R., Tse D., Baraze A. and Mill T. (1983) Photolysis of nitroaromatics in aquatic systems- 1 2,4,6-trinitrotoluene. Chemosphere 12, 316.

Mill T. Hendry D. G. and Richardson H. (1980) Freeradical oxidants in natural waters. Science $207,886-887$.

Moses F. G., Liu R. S. H. and Monroe B. M. (1969) The merry-go-round quantum yield apparatus. $\mathrm{Mol}$. Photochem. 1, 245-249.

Shackelford W. M. and Keith L. H. (1976) Frequency of organic compounds identified in water. EPA-600/4-76062. Athens, Ga

Spanggord R. J., Mill T., Chou T., Mabey W. R., Smith $J$. H. and Lee S. (1980) Environmental fate studies on certain munition wastewater constituents-literature review. SRI. Project No. LSU-7934 Final Report, Phase 1-Literature Review, SRI International, Menlo Park. Calif.

Zepp R. G. (1978) Quantum yields for reaction of pollutants in dilute aqueous solution. Envir. Sci. Technol. 12, 327-329.

Zepp R. G. (1982) Experimental approaches to environmental photochemistry. In The Handbook of Environmental Chemistry (Edited by Hutzinger O.), Vol. 2, Part B, pp. 19-41. Springer, Berlin.

Zepp R. G. and Baughman G. L. (1978) Prediction of photochemical transformation of pollutants in the aquatic environment. In Aquatic Pollutants. Transformation and Biological Effects (Edited by Hutzinger O.. Van Lelyveld I. H. and Zeeteman B. C. J.) p. 244. Pergamon Press, New York.

Zepp R. G. and Cline D. M. (1977) Rates of direct photolysis in aquatic environment. Envir. Sci. Technol. 11, 359366 .

Zepp R. G. and Schlotzhauer P. F. (1981) Comparison of photochemical behavior of various humic substances in waters---III. Spectroscopic properties of humic substances. Chemosphere 10, 479486.

Zepp R. G., Schlotzhauer P. F. and Sink R. M. (1985) Photosensitized transformations involving electronic energy transfer in natural waters: role of humic substances. Encir. Sci. Technol. 19, 74.81 